\title{
Erratum: Implementing the Fast Multipole Method in Three Dimensions ${ }^{1}$
}

\author{
K. E. Schmidt ${ }^{2}$ and Michael A. Lee ${ }^{3}$
}

Received November 18.1996

Equation $(11)$ in this paper contained a typographical error. The correct result is

$$
\begin{aligned}
& \sum_{n, \ldots, p \neq 0,0.0)} r_{m, p}^{-(l+1)} Y_{l m}\left(\Omega_{m, p}\right)=\sum_{m, \ldots, p \neq 0.0 .0} Y_{m m}\left(\Omega_{m, m p}\right) \frac{2^{\prime+2}}{\sqrt{\pi}(2 l+1) ! !} \\
& \times\left(\frac{I_{1}\left(\alpha r_{m o p}\right)}{r_{m o p}^{l+1}}-\frac{\alpha^{2 l+1} r_{m m p}^{\prime}}{2} \exp \left(-\alpha^{2} r_{m p p}^{2}\right)\right. \\
& \left.+\frac{(2 l+1) i^{\prime} \pi^{\prime-1 / 2} r_{n o p}^{\prime-2}}{4} \exp \left(-\frac{\pi^{2} r^{2}{ }_{n o p}}{\alpha^{2}}\right)\right)
\end{aligned}
$$

where $\mathbf{r}_{m(\eta)}=n \hat{\imath}+o \hat{y}+p \hat{z}$, and $n, o$, and $p$ are integers.

The conclusions and results of the paper are unaffected. A computer program subsequently distributed for the Fast Multipole Method has always been correct.

\footnotetext{
' This paper originally appeared in J. Stat. P/lys. 63:1223 (1991).

2 Department of Physics and Astronomy, Arizona State University, Tempe. Arizona 85287.

${ }^{3}$ Department of Physics. Kent State University, Kent. Ohio 44242.
} 